\title{
Educar para incluir
}

Antonio Pérez Esclarín ${ }^{21}$

(pesclarin@gmail.com)

Artículo recibido en agosto y aprobado en diciembre 2019

\section{Resumen}

La educación inclusiva tiene grandes desafíos ante un mundo absolutamente inequitativo y excluyente. Una educación para todos y todas asegurando su permanencia, la calidad educativa y la educación ética, ciudadana y compasiva son parte de los grandes desafíos de una educación verdaderamente inclusiva, cada uno de ellos incorpora tareas urgentes para generar cambios que gesten un mundo mejor.

Palabras clave: Educación inclusiva, calidad educativa, educación ética, ciudadanía, inclusión

\section{Abstract}

Inclusive education has great challenges posing by an absolutely inequitable and exclusive world. An education for all, thus ensuring that it will be continued, its educational quality and its ethical, citizen and compassionate education as part of the great challenges of a truly inclusive education. Each of them incorporates urgent tasks to generate changes towards a better world.

Key words: Inclusive education, educational quality, ethical education, citizenship, inclusion.

21 Doctor en Filosofía por la Pontifica Universidad Católica del Ecuador. Master in Divinity. Woodstock College of New York. Licenciado en educación por la Universidad Nacional Experimental Simón Rodríguez de Venezuela. Licenciado en Filosofía por la Pontificia Universidad Católica del Ecuador. Escritor de numerosos libros y ensayos. Profesor jubilado de la Universidad Simón Rodríguez. Formador del equipo del Centro de Formación e Investigación Padre Joaquín de Venezuela. 


\section{Introducción}

Resulta contradictorio que América Latina, el continente con mayor número de católicos, es el continente de mayor inequidad, con la más injusta distribución del ingreso. De hecho, si es innegable que en algunos países se tomaron políticas orientadas a erradicar la pobreza y el hambre, todavía abunda demasiado la miseria y resultan cada vez más escandalosas las diferencias entre el grupito de privilegiados y las mayorías que tienen serias dificultades para vivir dignamente o sencillamente vivir. Por ejemplo, América Latina produce alimentos para dar de comer adecuadamente a 1.800 millones de personas, es decir, a tres veces su población. Sin embargo, el 17\% de los niños están desnutridos y son millones los que pasan hambre. (Martínez, 2005)

Por otra parte, arropada por un discurso abiertamente inclusivo y justiciero, germinó con fuerza una corrupción desaforada que levantó muchos nuevos ricos que exhiben sin el menor pudor sus súbitas y malhabidas riquezas; otros siguen utilizando los recursos públicos como si fueran propios o los reparten como limosnas para ganarse las voluntades de las mayorías, con el creciente peligro de que las necesarias ayudas sociales terminen desestimulando el esfuerzo y contribuyan a reproducir la pobreza. Hoy, una gran parte vive de las ayudas sociales más que de los ingresos de sus trabajos y estos grupos pasan a ser "clientes" o "deudores" antes que ciudadanos y actores de la sociedad. Incluso, detrás de elocuentes discursos por una educación decididamente inclusiva, se puede ocultar la raíz de una exclusión más inmoral de todos aquellos que no siguen las políticas de los que mandan o se atreven a criticarlas. Puede resultar en verdad de un cinismo muy cruel y totalmente opuesto al sentido de la verdadera inclusión, incluir para formar sujetos excluidores, es decir, utilizar la educación, supuestamente inclusiva, para transmitir una ideología que impida a los educandos considerar la diversidad como riqueza y lleve a rechazar a los que no piensan como ellos o no disfrutan de las mismas condiciones económicas y sociales.

Lo que en América Latina más diferencia al núcleo con mayores ingresos de los demás es su perfil educativo. Los pobres están doblemente penalizados: a su condición de pobreza suman sus dificultades para el 
acceso y la permanencia en los centros educativos y la baja calidad de los servicios recibidos. No todos los alumnos llegan a la escuela en las mismas condiciones, ni todos reciben lo mismo de ella. La proclamada igualdad de oportunidades se convierte en un extraordinario mecanismo para mantener o agrandar las desigualdades. Los alumnos de familias privilegiadas gozan de ambientes familiares y de establecimientos educativos que estimulan sus autoaprendizajes, cuentan con padres cultos que les ayudan en sus tareas y tienen capacidad para exigir, entran desde muy temprano en contacto con los medios tecnológicos y culturales, asisten a un preescolar, van después a un colegio privado o a los públicos de mayor prestigio, que están bien dotados de recursos pedagógicos, y, por lo general, funcionan bien y en el que raramente se pierden días de clases, y hasta pueden contar con profesores particulares para adquirir refuerzos y superar sus deficiencias. De este modo, salen mucho mejor capacitados para acaparar los puestos más codiciados en las universidades públicas o en las privadas de mayor prestigio, con lo que luego obtienen los puestos de trabajo más prestigiosos y mejor remunerados, y de este modo se reproducen las desigualdades sociales.

Frente a las oportunidades de los alumnos de las familias más privilegiadas, los alumnos de las familias pobres, que tienen como principal objetivo asegurar la comida diaria, no suelen tener acceso en sus casas a los medios culturales y tecnológicos, con frecuencia sus padres son analfabetas o sólo cursaron los niveles educativos primarios, no van en su mayoría al preescolar, nivel y modalidad con creciente reconocimiento como factor esencial para una mayor equidad social y para un mejor desempeño educativo futuro de los alumnos. Incluso a muchos de ellos, la necesidad de trabajar o de conseguir comida cada vez más escasa, les va a impedir ir a la escuela. Los que lo hacen, son inscritos en escuelas por lo general, mal dotadas, con escasos recursos, en las que se suspenden clases por numerosos motivos: la falta de agua, el difícil acceso cuando llueve, la inseguridad, la ausencia de los maestros, o por asuntos gremiales. Los maestros, algunos de ellos verdaderos héroes, se sienten solos y muchos terminan desmotivándose por la falta de acompañamiento y estímulos, y tratan de emigrar a los centros educativos de mayor prestigio. Esta situación constituye un verdadero reto para la inclusión. 


\section{Desafíos de la educación inclusiva}

El problema de la inclusión es mucho más complejo de lo que se nos quiere hacer creer y supone enfrentar grandes desafíos. Una mirada rápida a la terrible inequidad que se vive en el continente y el mundo es una muestra de esa complejidad, ante esto presento algunos desafíos de la educación inclusiva:

\section{A.- Educación para todos(as) y permanencia en el sistema educativo}

La educación inclusiva es un derecho (Ainscow, M. y Echetta, G. , 2010). Es urgente seguir trabajando para que no haya un solo niño, niña o joven en América Latina sin acceso a la educación (Torres, 2000). Este desafío supone no sólo crear muchos nuevos centros educativos dignos y bien dotados, en especial de educación inicial que es donde existe el mayor déficit en la cobertura, sino también retenerlos en el sistema educativo el mayor tiempo posible para que no lo abandonen sin haber adquirido los valores y competencias esenciales para seguir aprendiendo y poder insertarse en la sociedad como ciudadanos productivos, honestos y solidarios. Esto va a suponer centros educativos al servicio de la diversidad de los alumnos, que traten de compensar las desigualdades de origen, y con una pedagogía activa, pertinente y productiva, para que los alumnos se sientan a gusto estudiando y palpen la utilidad y pertinencia de sus estudios.

Actualmente la educación secundaria es considerada un piso mínimo para salir de la pobreza, desarrollar la ciudadanía y tener mayores oportunidades de acceder al mundo laboral. La culminación de la secundaria es todavía una asignatura pendiente y está fuertemente condicionada por el origen social de los estudiantes. Es importante subrayar que este nivel educativo ya no garantiza la movilidad social porque cada vez son necesarios mayores años de estudio y niveles de conocimiento para acceder a los empleos más productivos. En algunos países ya se advierten señales de vulnerabilidad a la pobreza y la exclusión entre quienes completan la educación secundaria.

Enfrentar esta realidad va a exigir también la multiplicación de programas atractivos de capacitación laboral, emprendimiento y 
formación humana, dirigidos a los que abandonaron el sistema o fueron expulsados, que los dote de herramientas para incorporarse activamente en el mundo del trabajo y también puedan reinsertarse en el sistema de la educación formal. Pero estos programas alternativos deben ser de verdadera calidad, pues sería absurdo que los que acuden a ellos se vuelvan a encontrar con la misma metodología transmisiva y un ambiente desmotivador que estuvieron entre las causas principales que les llevó antes a abandonar los estudios.

\section{B.- La calidad educativa}

El derecho a la educación va mucho más allá del acceso, aunque es un primer paso, ya que exige que ésta sea de calidad y logre que todas las personas desarrollen al máximo sus múltiples talentos y capacidades. Lamentablemente, los avances en la cobertura no han ido acompañados de un mejoramiento sustantivo de la calidad de la educación, especialmente la que se ofrece a los estudiantes de contextos más desfavorecidos, por lo que su mayor acceso a la educación no ha supuesto necesariamente un mayor acceso al conocimiento que les permita acceder en igualdad de condiciones al mundo laboral y ser ciudadanos de pleno derecho. Las evaluaciones internacionales muestran los bajos niveles de aprendizaje en América Latina y su desigual distribución entre los diferentes grupos sociales. En consecuencia, el principal desafío en América Latina es avanzar hacia una mayor igualdad en la calidad, que garantice a todos los aprendizajes esenciales.

Hoy está de moda hablar de calidad, incluso de excelencia. Por ello, debemos comenzar aclarando qué estamos entendiendo por educación de calidad, dado que la concepción que predomina está atrapada en la lógica de la eficiencia, la producción y la rentabilidad. El lenguaje economicista y tecnocrático ha penetrado con fuerza en el sistema educativo y ha incorporado una mentalidad propia del mundo de la industria, el mercado, las empresas. Dicha mentalidad y su correspondiente terminología se metió en las políticas educativas, en el discurso de los docentes, en las instituciones de formación docente. Hoy se habla sin el menor pudor de recursos humanos o de capital humano, de insumos, de clientes (los alumnos y padres de familia), de gestión educativa, de empresas de servicios educativos, de mercado 
de productos pedagógicos, y hasta la palabrita competencia, hoy tan invocada y tan querida, nos viene del mundo empresarial. De hecho, las propuestas humanistas de calidad que suelen proclamarse, se diluyen a la hora de la verdad, donde se imponen los indicadores de rentabilidad y eficiencia que pocas veces miden las actitudes y valores, la calidad de las personas y los ciudadanos, ni toman en cuenta las diferencias de origen, recursos y posibilidades de los alumnos, ni su situación socioeconómica y cultural. De este modo, el aprender a aprender y el aprender a hacer se privilegian, aunque se niegue en las proclamas, sobre el aprender a ser, el aprender a convivir y el aprender a transformar. Cada vez más, los valores de justicia, equidad, dignidad humana, solidaridad y convivencia, van siendo sustituidos por la preocupación por la eficacia, por la competitividad, la búsqueda de resultados tangibles, el ajuste a las necesidades del mercado de trabajo y de la economía, la lucha por disponer de mejores condiciones de salida del sistema educativo ante un mundo laboral escaso, la formación de destrezas básicas, la necesidad de incorporar las tecnologías de la información y la comunicación, etc.

En consecuencia, es urgente que trabajemos por una concepción de calidad en torno a metas sociales, políticas, democráticas y humanistas. Educación orientada al desarrollo personal, social y espiritual de las personas. Educación que permita a cada persona y a todas las personas desarrollar a plenitud sus talentos y construirse como persona y como ciudadano productivo y solidario. Que le enseñe a ser, convivir, aprender, producir y también transformar este nuestro mundo inhumano para hacer realidad "Otro Mundo Posible" donde todos podamos vivir con dignidad.

En definitiva, para ser de calidad, la educación debe ligarse a la equidad, la justicia y la humanización. No podemos aceptar como de calidad una educación que discrimina, que excluye a los necesitados, que deja en el camino a los más débiles, que mantiene y cultiva la conciencia de superioridad. Cuando se habla del fracaso escolar, se señalan sobre todo los indicadores de lectura, escritura, matemáticas... Pero también fracasan los centros educativos que, aunque egresen alumnos con altísimas calificaciones y muy bien capacitados profesionalmente, no han logrado sembrar en ellos el compromiso de trabajar por el bienestar y la vida digna para todos. Se trata de formar personas plenas, 
honestas, respetuosas, sensibles y solidarias, y no meros profesionales exitosos. Por ello, la educación es de calidad sólo si egresa personas y ciudadanos de calidad.

Calidad para todos exige superar la "pedagogía de la pobreza", es decir, pensar que para los pobres basta con un currículo poco exigente, y egresar alumnos sin las competencias y saberes necesarios para seguir estudiando o ejercer una profesión adecuadamente. Esto va a suponer establecer o reforzar los diversos mecanismos compensatorios que permiten nivelar las condiciones de los estudiantes que sufren mayores dificultades para que puedan enfrentar sistemas de promoción que evalúen más homogéneamente, y con estándares más altos, las competencias hoy consideradas básicas para el desarrollo pleno de la ciudadanía social. Se trata, entonces, de nivelar hacia arriba, y no solo de retener en el sistema educativo y hacer egresar a los estudiantes a costa de una menor calidad y eficacia de los procesos de enseñanza. Esto significa, entre otras cosas, velar porque los procesos de promoción automática no se transformen en un desincentivo para el estudio y el esfuerzo. Pero no va a ser tarea fácil evitar el fracaso de los más débiles en un mundo que está organizado para reproducirlo. De ahí la necesidad de practicar la discriminación positiva, es decir, privilegiar y atender mejor a los que tienen más carencias y problemas, para así compensar en lo posible las desigualdades de origen y evitar agrandar las diferencias. Tratar a todo el mundo por igual en un mundo desigual, es favorecer más a los que más tienen, a costa de los que tienen menos. La discriminación positiva implica también que las escuelas cuenten con recursos especializados para atender a los alumnos con graves problemas de conducta y a los que tienen especiales dificultades de aprendizaje.

No puede ser que abandonen la escuela precisamente los que más necesitan de ella. En este sentido, Estado y sociedad deben aunar esfuerzos para que, en los centros educativos que atienden a los alumnos más necesitados, se les garantice a todos las mismas condiciones que tienen los alumnos de familias privilegiadas. Esto va a exigir, entre otras cosas, jornadas de trabajo más extensas y más intensas, dotación de buenas bibliotecas y utilización creativa de ellas, comedores escolares no como fines en sí mismos, sino como medios para favorecer el aprendizaje, salas tecnológicas y programas y capacitación adecuada de 
los docentes para que utilicen las nuevas tecnologías como recursos para el aprendizaje, talleres y laboratorios que favorezcan la pedagogía activa y la investigación, canchas deportivas amplias y buenos programas de educación física y deportes, lugares para estudiar e investigar con comodidad, actividades extraescolares atractivas. La experiencia demuestra que, para promover la calidad, no es suficiente la dotación de recursos (incluyendo los textos y las computadoras) o proporcionar alimentación a los alumnos sin una transformación de la pedagogía y una reorientación de las actividades y tiempos escolares. La inclusión va a exigir, sobre todo, trabajar para lograr los mejores maestros y profesores, con vocación de servicio, orgullosos de su profesión, con expectativas positivas de sí mismos y de cada uno de sus alumnos, motivados y que disfrutan enseñando, en formación permanente, ya no para engordar currículos, sino para desempeñar mejor su labor y servir con mayor eficacia a los alumnos, sobre todo a los más carentes y necesitados, capaces de impulsar una pedagogía que promueva la motivación, autoestima y deseos de aprender de sus alumnos.

Por todo esto, si en verdad se busca que la educación ayude a los pobres a salir de la pobreza, primero habrá que combatir la pobreza con políticas sociales eficientes y equitativas, y en segundo lugar habrá que sacar a la propia educación de la pobreza y combatir la pobreza económica y también pedagógica de numerosos educadores. Esto va a exigir aumentar la inversión en educación y en las políticas sociales que la posibiliten y favorezcan, de modo que se garantice la transparencia, la equidad y el buen uso. Y va a exigir revisar los procesos de formación de los docentes, tanto iniciales como permanentes, para convertirlos en verdaderos pedagogos y en educadores comprometidos en gestar una educación de verdadera calidad para todos.

Una educación inclusiva, orientada a garantizar el éxito de todos, debe tomar en consideración algunos elementos que, según varias investigaciones, son fundamentales para garantizarlo. En primer lugar, un fuerte liderazgo técnico pedagógico del equipo directivo. Necesitamos directivos expertos en humanidad y en pedagogía, promotores de entusiasmo, formación e innovación, para que sus educadores se vayan entendiendo como constructores de caminos y no como dadores de programas y caminadores de sendas abiertas por otros; 
como protagonistas de los cambios necesarios, como investigadores en la cotidianidad de las aulas y escuelas, lo que sólo es posible si se hace de la reflexión permanente, de la pregunta, del diálogo de saberes, una práctica habitual, si cada uno se asume más como aprendiz que como docente, lo que supone humildad, un estado de insatisfacción permanente y sobre todo alegría y disfrute.

Otro elemento clave para garantizar el éxito escolar lo constituyen las altas expectativas de los docentes sobre las posibilidades de aprendizaje de todos los alumnos: Si creen que tienen frente a sí un grupo de alumnos inteligentes, capaces, maravillosos, los tratan como a tales y logran que actúen de ese modo. Pero si creen que no saben nada, que son apáticos, brutos, imposibles, terribles, logran que los alumnos actúen según esta percepción negativa.

Un tercer elemento clave para el éxito escolar es un clima ordenado y seguro. Si en los centros escolares impera la violencia, el desorden, la indisciplina, va a ser muy difícil o incluso imposible que los alumnos aprendan lo que tienen que aprender. Para mejorar la calidad de las escuelas hay que hacer que los alumnos estén bien atendidos, se sientan bien en su escuela y aprendan de manera óptima lo que necesitarán para su vida en la sociedad. Necesitan pues una escuela en la que se viva en un ambiente agradable pero exigente, en el que los docentes y directivos garanticen un bienestar colectivo, en un clima de respeto mutuo, de tolerancia ante lo diverso y de no tolerancia ante la agresividad y la violencia.

Un cuarto elemento esencial para el éxito escolar es garantizar a todos los alumnos las herramientas esenciales para un aprendizaje autónomo y permanente: lectura personal y autónoma de todo tipo de textos y del contexto, de los nuevos lenguajes digitales y de la imagen; escritura, pensamiento lógico-matemático y científico, solución de problemas, ubicación en el espacio y en el tiempo; y de aquellas actitudes esenciales para un aprendizaje autónomo y permanente: curiosidad, reflexión, investigación, crítica y autocrítica, deseos de aprender y de hacer las cosas cada vez mejor, exigencia, esfuerzo, trabajo en equipo. 
Otros factores importantes para el éxito escolar, son la fuerte relación entre el centro educativo y las familias de los alumnos; la buena formación de los docentes y su preocupación por seguirse formando; el que trabajen en un solo plantel; el número de días de clase y el uso eficiente del tiempo en el aula, pues está bien comprobado que no sólo se pierde el tiempo cuando se suspenden las clases, sino que también se pierde, y mucho, dentro del aula cuando los alumnos están dedicados a hacer actividades rutinarias que no llevan a aprendizajes significativos.

La educación inclusiva va a exigir también una revisión profunda de la cultura y las prácticas de evaluación, que suele ser el principal mecanismo de los docentes para excluir a los alumnos. Hay que superar esa pedagogía que convierte la evaluación en un instrumento de control, sanción y exclusión del alumno y sirve para reforzar la distancia entre éste y el profesor, y de los alumnos entre sí.

La educación inclusiva busca el éxito y no el fracaso de los alumnos. Este es el criterio que debería guiar a la evaluación, criterio que, sin embargo, está muy lejos de las prácticas habituales. Hay docentes que llegan a enorgullecerse de su fracaso. No conozco ningún médico que vaya alardeando por allí de que, de cincuenta enfermos que atendió, sólo le sobrevivieron cuatro. Tampoco conozco ningún ingeniero que se ufane de que la mayoría de los edificios que empieza nunca quedan terminados o se derrumban pronto. Pero sí conozco educadores que exhiben sin el menor pudor su fama de "raspadores", y hasta se les oye comentar, sin pena, casi con gozo: "De cuarenta alumnos, sólo me pasaron siete". Ignoran que el único modo de comprobar la idoneidad de un docente es mediante el éxito de sus alumnos. Si los alumnos salen mal, él también está saliendo mal pues no logró motivarlos y guiarlos para que aprendieran lo que tenían que aprender. No olvidemos que toda evaluación que propone el docente se convierte en su propia autoevaluación a la luz de los resultados de sus alumnos.

Una educación inclusiva no culpa a los alumnos de su fracaso, pero tampoco lo oculta. No olvidemos que no se trata de que los alumnos "pasen de un grado a otro", para inflar los números de permanencia y prosecución, sino de garantizarles los aprendizajes necesarios. Puede ser un muy fuerte desestímulo a la calidad y al esfuerzo necesario que 
ella supone, promover a los alumnos sin los conocimientos esenciales necesarios. Si los alumnos no aprendieron, habrá que revisar el contexto y la experiencia de aprendizaje para ver qué está funcionando mal: el método, la motivación, los materiales, los conocimientos previos, las estrategias..., para introducir las modificaciones necesarias para que los alumnos tengan éxito y puedan ser promovidos justamente. La evaluación se convierte entonces en un medio excelente para que el profesor conozca cuáles son las fortalezas y carencias de cada alumno para así poderle brindar la ayuda que necesita.

Es, en consecuencia, muy necesario pasar de enseñar para evaluar después, a evaluar continuamente para enseñar mejor. Más que juzgar el pasado, la evaluación debe orientarse a preparar el futuro. Evaluación no para clasificar, castigar y excluir, sino para ayudar, para evitar el fracaso, para que, con la ayuda de todos, todos tengan éxito. Este tipo de evaluación inclusiva no castiga el error, sino que lo asume como una maravillosa oportunidad de aprendizaje. Si todos repetimos que "los errores enseñan", ¿por qué los castigamos? Si nos hacemos todos conscientes del error, estaremos poniendo las bases para superarlo.

\section{C.- Educación ética, ciudadana y compasiva}

La inclusión implica dotar a los alumnos de una sólida formación ética, ciudadana y compasiva para que se conviertan en incluidores de los propios compañeros con dificultades y les apoyen y ayuden, y trabajen por un mundo más igualitario y más justo, sin excluidos, donde erradiquemos de una vez la miseria y todos podamos vivir dignamente. Para ello, debemos alimentar la compasión. Pero no debemos confundir compasión con lástima. La compasión comparte el sufrimiento del otro: padece-con. La lástima no contempla verse en el lugar del otro. Por otra parte, frente al consumismo que, en el Siglo XXI, es el "verdadero opio del pueblo", debemos cultivar la austeridad y estilos de vida más sencillos, para que los bienes y recursos de la tierra alcancen a todos.

Queremos insistir en que si los centros educativos no logran egresar alumnos verdaderamente dispuestos a combatir todo tipo de exclusión y a trabajar por Otro Mundo Posible donde desaparezca la 
miseria y toda forma de discriminación, también han fracaso como centros de inclusión, y sobre todo, si vocean su carácter de cristianos, han fracasado en lograr que sus alumnos hagan suyos los valores del evangelio.

\section{Conclusiones}

Si la educación inclusiva y de calidad, es un derecho, es también un deber humano fundamental, lo que implica que todos somos corresponsables y debemos colaborar para que este derecho se cumpla. La defensa de los derechos humanos para todos se convierte en el deber de todos de hacerlos posibles. Afirmar, por ejemplo, el derecho de todos a una educación de calidad, implica que es nuestro deber esforzarnos y trabajar con ahínco para que todos los niños y jóvenes disfruten de la educación que queremos para nuestros hijos. Puede resultar de un gran cinismo proclamar derechos y mantener unas condiciones de vida que impiden su realización. Mientras no pasemos de los derechos a los hechos seguiremos aturdidos por una retórica complaciente y estéril. Estado, sociedad y también las familias, deben asumir su deber y responsabilidad educativa. También los Medios de Comunicación tienen una gran responsabilidad educadora y deben apoyar con su programación las políticas educativas orientadas a incluir a todos para formarlos como personas autónomas y ciudadanos responsables y solidarios.

La educación inclusiva y de calidad para todos, condición indispensable para la sana convivencia democrática, la productividad y el logro de instituciones eficaces, debería ser la estrategia fundamental de las familias, el Estado y la sociedad para incorporar plenamente a todas las personas al quehacer de la vida pública contemporánea. Si realmente estamos convencidos de que la educación inclusiva y de calidad para todos es exigencia para la dignidad y libertad de las personas, clave de la democracia política, del crecimiento económico y de la equidad social, debería ocupar el primer lugar entre las preocupaciones públicas y entre los esfuerzos nacionales. De ahí, la necesidad de asumir la educación inclusiva de calidad como tarea de todos, como proyecto nacional, y nunca de un partido o una parcialidad política, objeto de consensos sociales amplios y duraderos. 
Es evidente que al Estado le corresponde la más amplia responsabilidad: él debe liderar la puesta en marcha de un verdadero proyecto educativo, en coherencia con el proyecto de país que se quiere bosquejado en la Constitución, capaz de movilizar las energías creadoras, la participación activa y el entusiasmo de toda la sociedad, para combatir de frente la ignorancia, la mala educación, la exclusión, la ineficiencia, la corrupción, la retórica, la mediocridad. Hay que convertir discursos, proclamas y buenos deseos en políticas y concretarlas en acciones.

El Estado, que representa el interés común y ejerce un poder conferido por la sociedad, debe vigilar y garantizar que el derecho a la educación inclusiva y de calidad se cumpla en términos de equidad, lo que implica compensar las desventajas de los más pobres para que las diferencias de origen no se conviertan en desigualdades y se reproduzca la pobre oferta educativa para los más pobres.

\section{Bibliografía}

Ainscow, M. y Echetta, G. (2010). La educación inclusiva como derecho. Marco de referencia y pautas de acción para el desarrollo de una revolución pendiente. Granada.

Martínez, R. (2005). Hambre y desigualdad en los países andinos. La desnutrición y la vulnerabilidad alimentaria en Bolivia, Colombia, Ecuador y Perú. Cepal, ONU, serie 112. Recuperado en: https:// repositorio.cepal.org/bitstream/handle/11362/6116/1/S05788_ es.pdf

Torres, R.M. (2000). Educación para todos, una tarea pendiente. Educación Popular, Madrid. 